\title{
Foundations of Psychiatric Sleep Medicine
}

\author{
David T. Plante and John W. Winkelman, editors; Cambridge University Press: New York, NY, 2010; \\ 415 pages; ISBN 978-0-521-51511-5; \$99 \\ Frank M. Ralls, M.D. ${ }^{1,3}$; Swala K. Abrams, M.D. ${ }^{2,4}$ \\ ${ }^{1}$ Division of Pulmonary, Critical Care, and Sleep Medicine, Department of Internal Medicine and the ${ }^{2}$ Department of Psychiatry, \\ University of New Mexico School of Medicine, Albuquerque, NM; the ${ }^{3}$ University of New Mexico Hospital Sleep Disorders Center, \\ Albuquerque, NM; and the ${ }^{4}$ University of New Mexico Hospital Psychiatric Center COPE Clinic, Albuquerque, NM
}

$\mathrm{T}$ he subject of mental illness makes some physicians uncomfortable, and as such, many avoid this group of patients entirely or are unable to see beyond the most obvious symptoms of the psychiatric illness. One example of this is the schizophrenic patient presenting to the Emergency Department (ED) in the early morning with a chief complaint of "I can't sleep because the devil won't stop pounding on my chest. I know he is trying to steal my heart!" Once identified as mentally ill, this patient may be quickly transferred to the psychiatric ED without the realization that he was complaining of cardiac chest pain but expressing the symptom in a manner consistent with his thought disorder; what may be missed is a devastating ischemic event. Specifically in terms of the practice of sleep medicine, the importance of sleep in the psychiatric patient is too often vastly underestimated and misunderstood. While the effects of psychiatric illness on sleep symptomatology are commonly taught, the concept that poor sleep may greatly exacerbate mental illness frequently is not given the attention it deserves in medical school and beyond. Poor sleep will often precipitate manic or psychotic decompensation and cause otherwise treatable depression to continue as a smoldering illness. Consequently, the sleep medicine physician must develop an appreciation for the bidirectional interactions between sleep/wake disorders and mental illness. More broadly, a basic understanding of sleep physiology and how sleep disorders (particularly insomnia) affect the mind are essential to every practicing provider. Sleep disorders that are not properly diagnosed can lead to significant economic impact and a loss of individual and family quality of life, and the additional morbidity associated with untreated sleep pathology in the psychiatric patient can be profound.

Foundations of Psychiatric Sleep Medicine consists of 24 chapters written by a host of well-known experts in the fields of sleep medicine and psychiatry. The text begins by providing historical context and then, chapter by chapter, gently takes the reader through basic elements of sleep physiology, the extremely important topic of how to take a sleep medicine history, and discussions of common and uncommon sleep disorders, thereby expanding the reader's knowledge one step at a time. The editors should be commended for including more obscure disorders such as Kleine-Levin Syndrome and the neurodegenerative synucleinopathies associated with REM sleep behavior disorder (RBD): dementia with Lewy bodies (DLB), Parkinson disease (PD), and multiple system atrophy (MSA). It is profoundly refreshing to read a book that incorporates significant diseases that affect the geriatric population such as these neurodegenerative disorders. On the other end of the age spectrum, the authors give excellent, detailed insight into the role of psychiatry in pediatric sleep disorders including insomnia, developmental disorders such as Down syndrome (DS) and Prader-Willi syndrome (PWS), bipolar disorders, and schizophrenia.

The authors do a splendid job of providing the visual learner with excellent graphs, tables, and pictures. Examples include a sleep $\log$, sleep diary, and a table of commonly used medications along with class of drug, half-life, mechanism of action, and common side effects. The book is careful to address a common modern day problem affecting sleep: substance use disorders that go beyond caffeine and nicotine to include cocaine and amphetamines.

In our roles as educators, we often encounter residents in internal medicine and in psychiatry who are eager to learn some sleep medicine but find that the major textbooks in this area are too long to read in the limited amount of time available during a sleep medicine rotation. In contrast, this book is succinct, up to date, well written, and filled with useful clinical information that can be used immediately by the busy health professional.

In summary, Foundations of Psychiatric Sleep Medicine starts the reader on an adventure that begins with the very first page and does not end until all 415 pages of informative and well-written text are fully digested. We can easily imagine this book finding a place on the bookshelf (or the first time through, the night stand or next to a favorite reading chair) of every physician interested in psychiatry, sleep, and the many interactions between these two disciplines.

\section{CITATION}

Ralls FM; Abrams SK. Foundations of psychiatric sleep medicine. J Clin Sleep Med 2013;9(5):521-522. 


\section{SUBMISSION \& CORRESPONDENCE INFORMATION}

Submitted for publication January, 2013

Accepted for publication January, 2013

Address correspondence to: Frank M. Ralls, M.D., Assistant Professor of Internal Medicine, Medical Director, Adult Sleep Medicine Services and Program Director,

Fellowship in Sleep Medicine, University of New Mexico Hospital Sleep Disorders Center, 1101 Medical Arts Avenue NE, Building \#2, Albuquerque, NM 87102; Tel: (505) 272-6110; Fax: (505) 272-6112; E-mail: fralls@salud.unm.edu

\section{DISCLOSURE STATEMENT}

The authors have indicated no financial conflicts of interest. 